\title{
Optimization of DC-DC Converters for Improved Electromagnetic Compatibility With High Energy Physics Front-End Electronics
}

\author{
C. Fuentes, B. Allongue, G. Blanchot, F. Faccio, S. Michelis, Student Member, IEEE, S. Orlandi, \\ J. Pontt, Senior Member, IEEE, J. Rodríguez, Senior Member, IEEE, and M. Kayal
}

\begin{abstract}
The upgrade of the Large Hadron Collider (LHC) experiments at CERN sets new challenges for the powering of the detectors. One of the powering schemes under study is based on DC-DC buck converters mounted on the front-end modules. The hard environmental conditions impose strict restrictions to the converters in terms of low volume, radiation and magnetic field tolerance. Furthermore, the noise emission of the switching converters must not affect the performance of the powered systems. A study of the sources and paths of noise of a synchronous buck converter has been made for identifying the critical parameters to reduce their emissions. As proof of principle, a converter was designed following the PCB layout considerations proposed and then used for powering a silicon strip module prototype for the ATLAS upgrade, in order to evaluate their compatibility.
\end{abstract}

Index Terms-Air-core Inductors, DC-DC buck converter, EMI, power distribution.

\section{INTRODUCTION}

$\mathbf{T}$ HE Large Hadron Collider (LHC) at CERN is planned to be upgraded in the future to increase its luminosity. To cope with this, the resolution of the sub-detectors within each High Luminosity LHC (HLLHC) experiment must be improved through an increase of the number of sensors. Therefore the power consumption of the Front-End Electronics (FEE) will rise as well. The present powering scheme, based on DC power supplies located in radiation-free areas, approximately 100 meters far from the detectors, can not be used for the HLLHC due to the lack of space for running more power cables and its poor efficiency. One possible solution [1] under study is based on on-detector DC-DC converters [2], which must be radiation [3] and magnetic field [4] tolerant. Furthermore, the converters must be as small as possible and must emit the lowest electromagnetic

Manuscript received December 20, 2010; revised April 07, 2011; accepted June 05 , 2011. Date of publication July 22, 2011; date of current version August 17, 2011. C. Fuentes was supported by MECESUP-Chile under Grant FSM0601.

C. Fuentes is with CERN, 1211 Geneva 23, Switzerland and also with UTFSM, 110v Valparaiso, Chile (e-mail: cristian.alejandro.fuentes.rojas@ cern. ch).

B. Allongue, G. Blanchot, F. Faccio, and S. Orlandi are with CERN, 1211 Geneva 23, Switzerland.

S. Michelis is with CERN, 1211 Geneva 23, Switzerland and also with EPFL, 11015 Lausanne, Switzerland.

J. Pontt and J. Rodríguez are with UTFSM, 1110v Valparaiso, Chile.

M. Kayal is with EPFL, Lausanne 11015, Switzerland.

Color versions of one or more of the figures in this paper are available online at http://ieeexplore.ieee.org.

Digital Object Identifier 10.1109/TNS.2011.2159395
(EM) noise in order to power the sensitive FEE without perturbing their performance.

Switching converter topologies as the DC-DC Buck allow the size reduction up to the desired level, but unfortunately they intrinsically generate electromagnetic interferences (EMI). On the other hand, these converters need an inductor for their operation, which must be air-core as any ferromagnetic material would saturate at the levels of magnetic field present in the LHC experiments (up to 4 Tesla). Due to the size constrain, the air-core inductor value can not exceed $500 \mathrm{nH}$. Therefore, for nominal input and output voltages of $10 \mathrm{~V}$ and $2.5 \mathrm{~V}$ respectively, the converter switching frequency must be of the order of few (1-4) $\mathrm{MHz}$ for reducing the inductor current ripple to an acceptable value of 1-4 A (1). These constrains limit the noise reduction strategies.

This paper describes the design of a low noise DC-DC Buck converter able to power the sensitive electronics of the HLLHC experiments without deteriorating their performance. First, the EMI sources of a buck converter topology are identified and estimated. Afterwards, the impact of non-idealities of components and PCB traces are evaluated to see their repercussion on the noise emitted by the converter. Electromagnetic couplings between components are taken into account and strategies for reducing their impact through segregation and correct orientation of the passives are proposed. Finally, these design strategies are implemented in a buck converter prototype which is used for powering an ATLAS short-strip tracker module prototype. This system test demonstrates the feasibility of the use of a buck converter for powering FEE at the upgraded LHC experiments.

\section{IDENTIFICATION OF THE CONVERTER'S SOURCES OF NOISE AND SELECTION OF THE INDUCTOR VALUE}

The reduction of the converter's emissions requires the identification and understanding of the sources of noise. They are originated by means of the commutation and can be found through the analysis of the converter's operation. The basic schematic of a synchronous Buck converter is shown in Fig. 1, where $S_{w 1}$ and $S_{w 2}$ are the two switches. Their alternating operation over a period $(\mathrm{T})$ produces a rectangular voltage in the phase node $\left(V_{S_{w 2}}\right)$. Its amplitude is equal to $V_{i n}$ during $S_{w 1}$ conduction time $\left(t_{o n}\right)$ and to zero otherwise (Fig. 2(a)). $V_{S_{w 2}}$ is then filtered by a low pass LC filter, producing an output DC voltage equal to the average value of the rectangular signal $\left(V_{i n} \cdot t_{o n} / T\right)$. The inductor current has a triangular shape, 




Fig. 1. Basic schematic of a synchronous Buck DC-DC converter including the main signals. Currents in red, voltages in black.

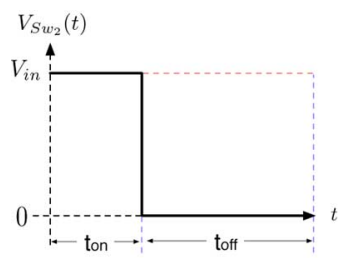

(a)

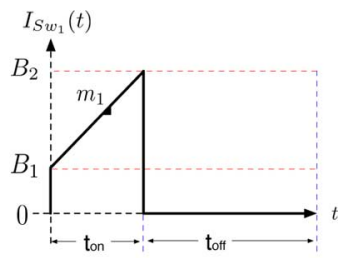

(b)
Fig. 2. Voltage and current sources. (a) Phase node voltage. (b) $S_{w 1}$ current.

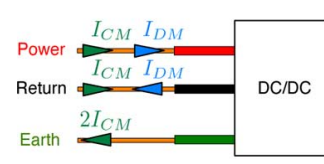

(a)

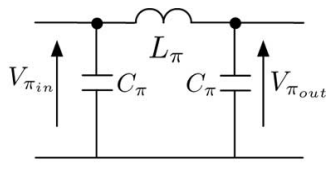

(b)
Fig. 3. Definition of CM and DM currents. Low pass $\pi$ filter for reducing DM conducted noise. (a) CM and DM definition. (b) Differential mode $\pi$ filter.

corresponding to the sum of the currents through $S_{w 1}$ and $S_{w 2}$ (Fig. 1). Its peak to peak value $\left(I_{L_{p p}}\right)$ can be defined as:

$$
I_{L_{p p}}=m_{1} \cdot t_{o n}=\frac{\left(V_{\text {in }}-V_{\text {out }}\right)}{L} \cdot \frac{V_{\text {out }}}{V_{\text {in }}} \cdot T
$$

and its minimum and maximum are $B_{1}=I_{o u t}-I_{L_{p p}} / 2$ and $B_{2}=I_{\text {out }}+I_{L_{p p}} / 2$, respectively. A typical $I_{S_{w 1}}$ current is shown in Fig. 2(b).

The AC content of the signals exiting the converter corresponds to the EM noise emitted. The latter is said to be "conducted" if propagated along the cables and "radiated" if propagated through the air. The conducted current can be decomposed into two auxiliary currents, known as differential mode (DM) and Common mode (CM). The DM current $\left(I_{D M}\right)$ corresponds to the functional current for the converter, like $I_{S_{w 1}}$. It enters and leaves the converter through the power and return cables with the same magnitude but opposite direction (Fig. 3(a)). The DM current can be reduced adding $\pi$ filters like the one of Fig. 3(b) at the input and output of the converter. On the other hand, CM current $\left(I_{C M}\right)$ enters with the same magnitude through the power and return cables (Fig. 3(a)) and returns through earth with twice the magnitude. The CM current is not intended to be present, but it will be in any practical system. Common Mode chokes are normally used to decrease $I_{C M}$, but can not be used in our application as ferromagnetic materials saturate in the magnetic field. Another way for decreasing $I_{C M}$ must be found.



Fig. 4. Amplitude of the first ten harmonics of $I_{S_{w 1}}$ as a function of the inductor value, for a given load ( $2 \mathrm{~A})$ and frequency $(3 \mathrm{MHz})$.

All the signals in the converter, including the noise, are originated by the $S_{w 1}$ current, the phase node voltage and their interaction with the passive components.

The AC part of $I_{S_{w 1}}$ and $V_{S_{w 2}}$ are identified as the noise sources of the converter. Their amplitude can be analyzed in the frequency domain by means of complex Fourier series, that decompose a periodic signal in an infinite sum of sinusoidal signals whose frequencies are integer multiples of the switching frequency $f_{s}(1 / \mathrm{T})$. Each sinusoidal signal of frequency $n \cdot f_{s}$ is said to be the harmonic signal of order $\mathrm{n}$; the harmonic of frequency $f_{s}(n=1)$ is said to be the fundamental. The amplitude of the n-th harmonic of $V_{S_{w 2}}$ is equal to $V_{n}$ as shown in (2). Similarly, the magnitude of the $\mathrm{n}$-th harmonic of $I_{S_{w 1}}$ is equal to $I_{n}$, as in (3).

$$
\begin{aligned}
V_{n} & =\left|\frac{2 V_{i n}}{j n \omega_{s} T}\left(1-e^{-j n \omega_{s} t_{o n}}\right)\right| \\
I_{n} & =\mid \frac{2}{j n \omega_{s}}\left[\frac{m_{1}}{j n \omega_{s} T}\left(1-e^{-j n \omega_{s} t_{o n}}\right) \cdots\right. \\
& \left.+\frac{B_{1}}{T}-\frac{B_{2}}{T} e^{-j n \omega_{s} t_{o n}}\right] \mid .
\end{aligned}
$$

The amplitude of the noise voltage source (2) depends exclusively of $V_{i n}$ and the duty cycle $\left(t_{o n} / T\right)$. The rise and fall times of a real phase voltage will produce lower amplitude at high frequency harmonics compared to those of (2) where they were assumed zero as a worst case consideration. This voltage noise is filtered by the output LC filter of the converter. If a reduction of the inductor is needed for decreasing the mass in the experiment, the filter performance may be kept unchanged augmenting the capacitance accordingly.

On the other hand, the noise current source (3) depends on the inductor value $\mathrm{L}$ (due to $m_{1}, B_{1}$, and $B_{2}$ ). The reduction of the noise emitted by this source must be achieved by means of the reduction of each of the harmonic currents as established by (3). The magnitude of the first ten harmonics was computed for different inductance values for the particular case of $V_{i n}=10 \mathrm{~V}$, $V_{\text {out }}=2.5 \mathrm{~V}, f_{s}=3 \mathrm{MHz}$, and output load of $2 \mathrm{~A}$ (Fig. 4). It indicates that, for a given output current, the inductor value must be increased in order to reduce the input current noise amplitude. Unfortunately, size and material budget constrains limit 




Fig. 5. Amplitude of the first three harmonics of $I_{S_{1-1}}$ as a function of the inductor value, for a given load (2 A) and frequency ( $3 \mathrm{MHz})$.

the usable inductance volume, hence value, in our application. Therefore, the choice of inductor represents a compromise between material budget and noise degradation. Fig. 5 shows how the amplitude of the first three harmonics changes as a function of the inductance. From the noise point of view it seems not worth to increase the inductance above $500 \mathrm{nH}$ (sacrificing volume and material). On the other hand, a decrease of the inductance below $500 \mathrm{nH}$ reduces its size/volume at the penalty of more input current noise. For instance, decreasing the inductor from $500 \mathrm{nH}$ to $250 \mathrm{nH}$ results in a magnitude increase of $1 \%$ in the fundamental and $18 \%$ in the third harmonic. This increase is considered acceptable as this translates into an increase of maximum $10 \mathrm{~mA}$ per harmonic (which can be considerably reduced by the addition of a $\pi$-filter). In summary, the useful value of inductor for our application varies from $250 \mathrm{nH}$ to $500 \mathrm{nH}$. Along this paper, as the noise is tried to be minimized, a $500 \mathrm{nH}$ inductor is going to be used.

\section{IDENTIFICATION OF CRITICAL PARASITICS}

\section{A. Careful Selection of Components}

The mitigation of the noise emitted by the converter must be firstly achieved minimizing the loops of the switching currents at the board layout level. Beyond this point, a further reduction of the emitted noise can be obtained by the correct selection of passive components. For instance, the performance of a filter can be dramatically reduced due to the non-idealities of its components, where special attention must be paid in minimizing the equivalent series inductance (ESL) of capacitors.

Fig. 6 shows how the insertion loss of a $\pi$ filter (Fig. 3(b)) can be impaired by the increase of the capacitor's ESL. Furthermore, the magnetic coupling between components (like the coupling between the two capacitors) can affect the performance [5] as discussed in Section IV. Special attention in the placement of the capacitor must be paid as well, as the trace inductance contributes to the deterioration of the filter performance.

A converter with a schematic like the one of Fig. 7 has been manufactured using SMD (Surface Mount Devices) ceramic capacitors. The two switches have been integrated inside an application-specific integrated circuit (ASIC) (red dashed box), together with the PWM control circuitry (not shown in Fig. 7) [6] that provides the appropriate driving signals to $S_{w 1}$ and $S_{w 2}$.

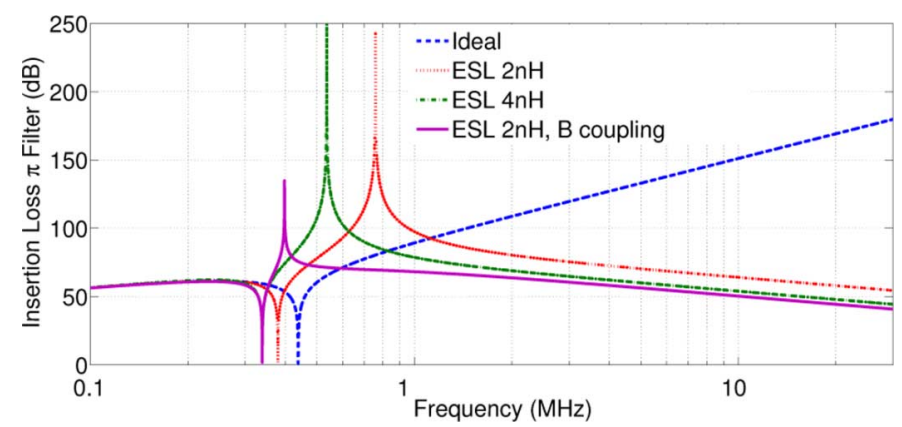

Fig. 6. Insertion loss of a $\pi$-filter for different capacitor's ESL values. The attenuation decreases as ESL augments. The filter performance can be further impaired if the two $\pi$-filter capacitors are magnetically coupled.

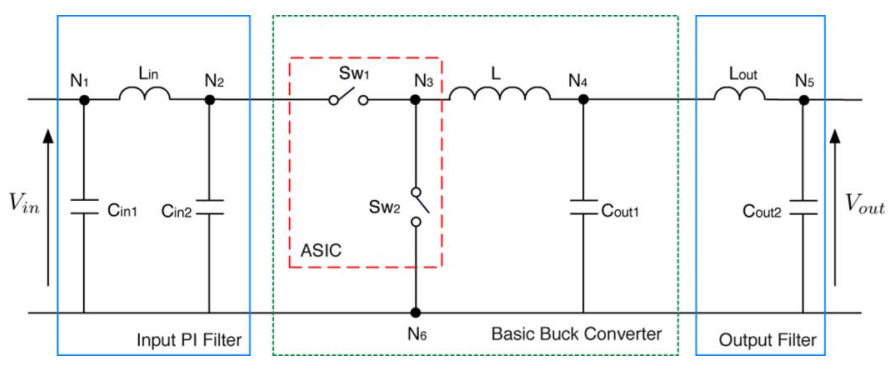

Fig. 7. Simplified schematic of a buck converter prototype. The two switches have been integrated inside an ASIC (red dashed box). Input an output filters (blue solid boxes) have been added to reduce the noise emissions.

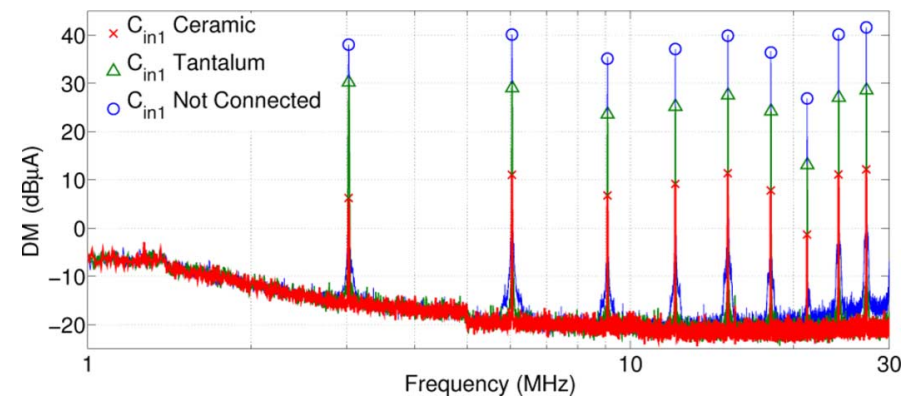

Fig. 8. DM input current for $C_{i n 1}$ with different ESLs.

The basic buck (dotted green box) has been enhanced through the addition of input and output filters (blue solid boxes) for reducing the DM switching noise. To evaluate the impact of the capacitor's non-idealities, $C_{i n 1}$ of the input filter has been replaced by a tantalum capacitor of the same value, but higher ESL. As expected, the input DM noise increases considerably approaching the value of noise that would be obtained if the capacitor were removed (Fig. 8). The selected tantalum capacitor had a 6 times higher ESL than the ceramic one, explaining the difference of noise at higher frequency. Similarly, the stronger difference at lower frequency (principally for the fundamental) is due to the larger equivalent series resistance (ESR) value of the tantalum capacitors.

Low ESL is therefore required to achieve low noise emissions. Low ESL capacitors are commonly available in the market place, within a given range of values and voltage ratings. To obtain larger capacitance values with further reduced ESL, other strategies as putting two capacitors of the same 


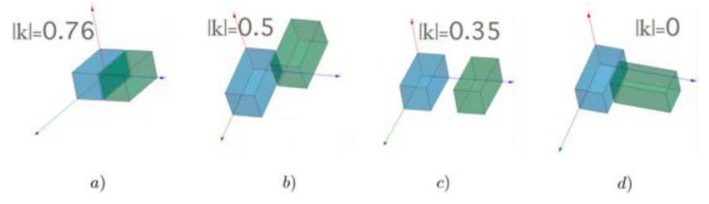

Fig. 9. Values of $k$ (coupling coefficient) for different positions of parallel capacitors.

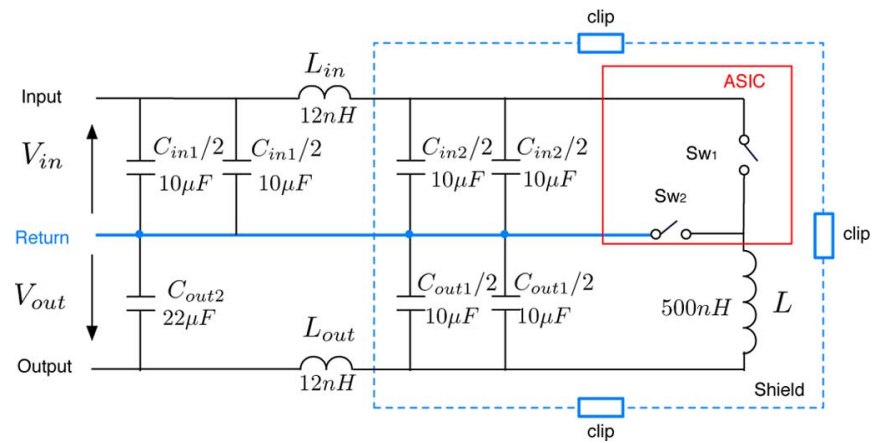

Fig. 10. Schematic of a buck with input and output filters. $C_{i n 1}, C_{i n 2}$ and $C_{\text {out } 1}$ consisted each of two capacitors of $10 \mu \mathrm{F}$ in parallel.

value in parallel can be used instead, [7] leading to an overall equivalent impedance of:

$$
Z_{e q}=\frac{1}{j w(2 C)}+j w \underbrace{\frac{(E S L+M)}{2}}_{L_{e q}}+\frac{E S R}{2},
$$

where $M=k \sqrt{E S L \cdot E S L}$ is the mutual inductance between the ESLs of the capacitors and $k$ is the coupling coefficient $(-1 \leq k \leq 1)$. For decreasing the overall inductance, the capacitors must be placed in such a way that their currents (equal in magnitude) oppose and therefore their magnetic fields subtract (M negative). Ideally, this equivalent inductance $\left(L_{e q}\right)$ could be made zero if the capacitors ESL are equal and tightly coupled $(|k|=1)$. Nevertheless, in a real placement, the $k$ coupling factor between capacitors is always smaller than 1 . Fig. 9 shows the coupling coefficient for two capacitors placed in different positions. For having a good coupling between them, they should be placed close together as in Fig. 9(a), while for decreasing the coupling it is more effective to place them orthogonally rather than to augment their relative distance (Fig. 9(c) and (d)). Using parallel capacitors for the input and output filters of Fig. 7, the prototype illustrated in Fig. 10 was produced. The capacitors $C_{i n 1}, C_{i n 2}$ and $C_{o u t 1}$ consisted each of two capacitors of $10 \mu \mathrm{F}$ in parallel. Each pair of capacitors was placed to achieve a magnetic coupling of about 0.5 (Fig. 9(b)). First, the capacitors were put such that their current flowed in opposite directions and their magnetic fields subtracted ( $\mathrm{M}$ negative). The input differential mode current for this configuration is compared in Fig. 11 to the case where the current in the 2 capacitors flowed in the same direction (M positive). The noise difference was up to $18 \mathrm{~dB}$.

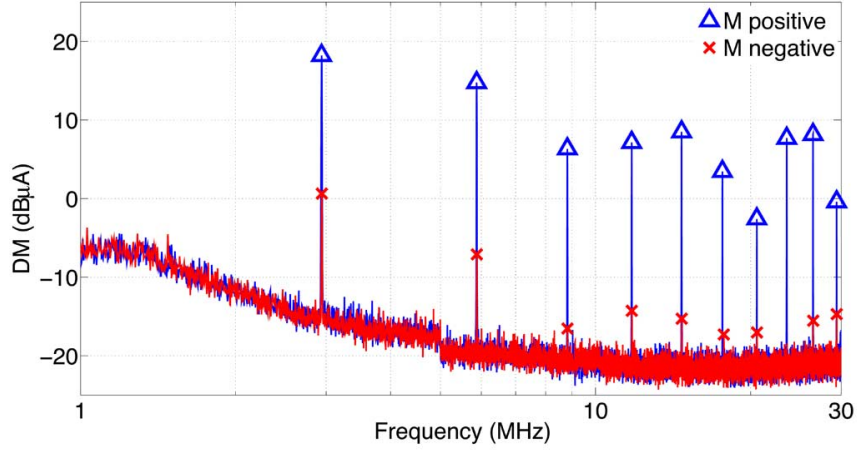

Fig. 11. DM input current for converter with parallel capacitors compared for positive and negative mutual inductance.

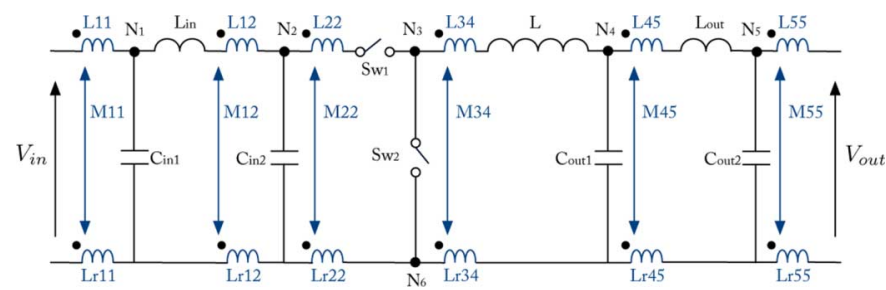

Fig. 12. The basic schematic of a synchronous Buck DC-DC converter is modified to include the inductance of the traces and return path plane.

\section{B. Return Path Bouncing}

The switching current $\left(I_{S_{w 1}}\right)$, mainly provided by the decoupling capacitors, must return to the source through the return layer of the Printed Circuit Board (PCB) where the converter is built. This return plane is not a zero impedance path [8], and therefore the time-varying currents will produce a voltage drop across the plane.

Fig. 12 illustrates the self partial and mutual inductances of the traces and return path plane of a buck converter. The values of these parasitic inductances were estimated through a 3D parasitic extraction software (Ansoft Q3D) and then introduced in a circuit simulator for a parametric simulation (Ansoft Simplorer). Simulation shows that the net partial inductance associated to the piece of plane where $I_{S_{w 1}}$ returns to $C_{i n 2}\left(L_{r 22, \text { net }}=\right.$ $L_{r 22}-M_{22}$ ) has a big impact on the common mode noise of the board. The lower this inductance, the lower the CM current and the magnetic field radiation. To reduce it, the decoupling capacitor should be placed as close as possible to the upper side switch $\left(S_{W 1}\right)$ and/or the PCB dielectric should be made thinner to increase the mutual inductance $M_{22}$.

The voltage drop in the parasitic inductance of the return path plane contributes to the deterioration of the $\mathrm{CM}$ noise, as it sets a voltage difference between the return nodes of the input and output connectors of the board. As it is impossible to make this inductance zero, a way of mitigating its impact is to place the input/output connectors close together and the ASIC further apart.

Two different PCB boards were designed based on the same schematic and components. They differed for the relative position of their input and output connectors that were placed either close to each other (Fig. 13(a)) or further away (Fig. 13(b)) (the inductor is removed to show the PCB). The configuration with 


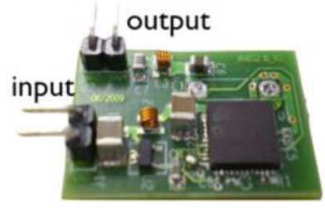

(a)

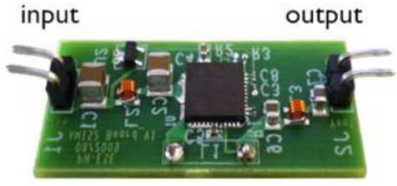

(b)
Fig. 13. Two converters with the same schematic and components but different placement of their input and output connectors. (a) Connectors put close to each other (b) Connectors put far from each other.



Fig. 14. CM noise comparison between converters of Fig. 13(a) and (b). The former shows a lower noise achieved through the reduction of the voltage bouncing between the return nodes of the input and output connectors (as they are placed closer together and far from the voltage drop across $L_{r 22, n e t}$ ).

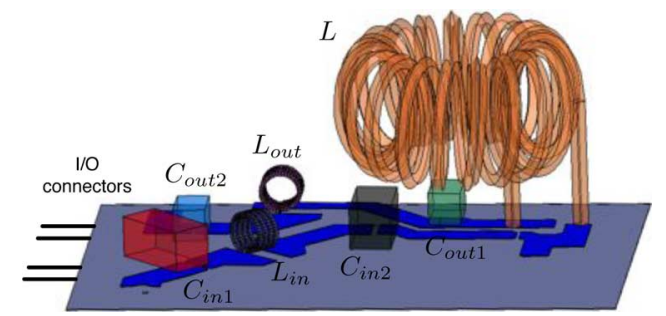

Fig. 15. Converter's 3D model used for the parasitic extraction software.

close connectors largely outperforms the other by up to $18 \mathrm{~dB}$ for the fundamental frequency (Fig. 14).

\section{IDENTIFICATION OF NOISY COMPONENTS}

As the size of the board is reduced, the couplings between the on-board passive components increase. While the magnetic coupling between some components can be useful to decrease the noise (Section III-A showed that for parallel capacitors), in other cases it can deteriorate the performance of the filters since a noisy component might couple magnetically its noise to other neighbors. To avoid this effect, noisy components must be identified [9] and segregated [10].

With a 3D parasitic extraction software (Q3D) the mutual inductance between passive components of a converter (Fig. 15) were determined. Their values were then swept within a possible range through a parametric simulation to identify their impact on the overall noise performance.

The AC component of $I_{S_{W 1}}$ current is mainly provided by $C_{i n 2}$ whilst the triangular current of the inductor $L$ flows in $C_{\text {out } 1}$. These largely varying currents radiate a magnetic field

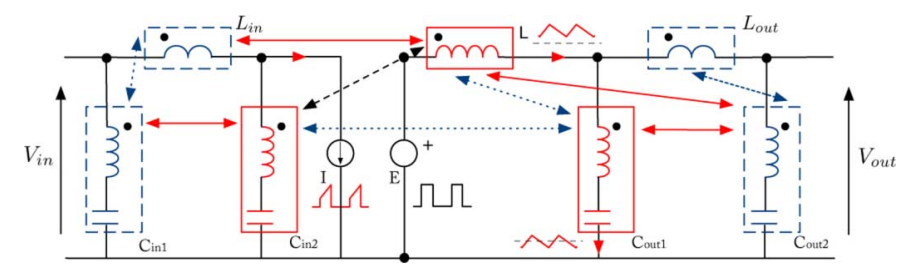

Fig. 16. Noisy (solid box) and quiet (dashed box) components.

that should not couple. These noisy components are highlighted inside red solid boxes in Fig. 16.

On the other hand, $C_{i n 1}, C_{\text {out } 2}, L_{\text {in }}$ and $L_{\text {out }}$ have much less current variations in time and can be seen as "quiet" components. In the event of noise coupling to any of those quiet components the noise is directly transferred at the output in the absence of any other filtering stage. Other than quiet, they are hence also susceptible components.

Through parametric simulations of the values obtained from the 3D parasitic extraction software, it was observed that couplings between noisy components could be tolerated, even though couplings between $L$ and $C_{i n 2}$ can lead to an increase of the input noise (black dashed arrow Fig. 16).

Couplings between noisy and quiet components (illustrated as red solid arrows $\longleftrightarrow$ in Fig. 16) must be avoided. Finally, couplings between quiet components (blue dotted arrows) do not have a strong impact, but it is preferable to isolate the quiet components of the output $\pi$ filter to achieve the lowest output noise.

\section{A. Segregation}

Components identified as noisy should be isolated from the susceptible components and from the connectors linking the board with the outside world (power supply, load). An example is shown in Fig. 15 where $C_{i n 2}, C_{\text {out } 1}, L$ have been isolated to the right end of the board.

Avoiding unwanted mutual inductance between components is better achieved by orthogonal placement (Fig. 9(d)) rather than distance (which also translates in larger boards). If perpendicular orientation is not possible, placement with some angle (as $C_{i n 1}$ at $45^{\circ}$ in Fig. 15) also helps. Of course the reduction of ESL of each capacitor also contributes to lower mutual inductances. However, it is impossible to avoid all the magnetic field interactions and in particular those originated by the varying current in the large main coil $L$. The choice of an appropriate inductor topology minimizing the emissions is mandatory.

\section{B. Selection of the Main Inductor Topology}

The main inductor is vital for filtering the rectangular phase node voltage and providing a DC voltage at the converter's output. Due to its triangular current, the inductor represents one of the main sources of magnetic field of the converter. Therefore, it is important to select an air core topology with enclosed AC magnetic field for not perturbing the sensitive FEE (nor the converter itself).

Air-core solenoid inductors are largely available in the market place. A $150 \mathrm{nH}$ commercial solenoid of $8 \mathrm{~mm}$ long and $4 \mathrm{~mm}$ of diameter was simulated for $1 \mathrm{~A}$ of current with Ansoft Maxwell 


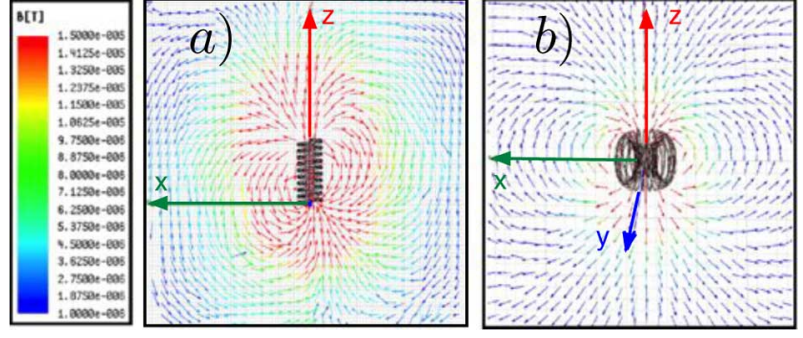

Fig. 17. Inductor topology comparison. Magnetic field simulated by means of a field solver simulator. (a) Solenoid (b) Toroid.

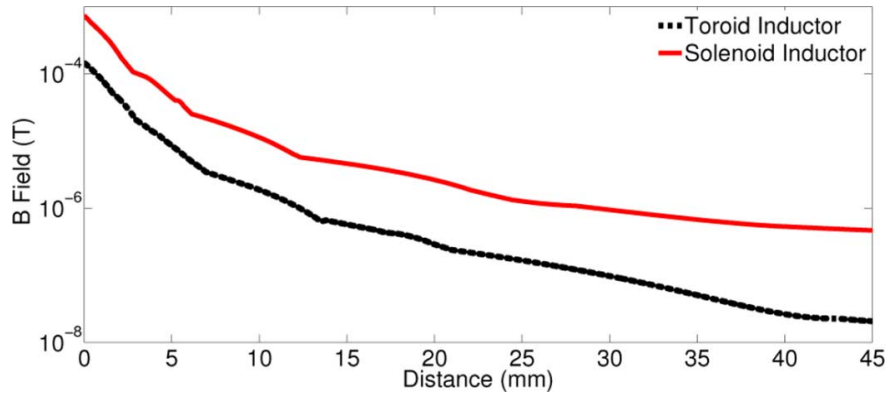

Fig. 18. Comparison of the magnetic induction of the solenoid and toroid along their $Z$-axis.

3D. A low value of inductor was chosen in order to reduce the computation time. The simulated magnetic field (Fig. 17(a)) shows that the field lines are not only concentrated inside the coil but also all around it, with non-negligible levels within 3 $\mathrm{cm}$ in all directions. This intense and widespread magnetic field emission represents the main drawback of such inductors. To reduce their emission, a conductive shield surrounding the inductors can be added. Nevertheless, it must be oversized in order not to affect the inductance value, representing another drawback of the solenoid.

Toroidal inductors are known for having a better contained magnetic field. Ferromagnetic-core toroids are widely used as power inductors, contrary to their air-core counterparts. In order to evaluate them, custom air-core toroids have been produced. A custom $200 \mathrm{nH}$ air-core inductor of diameter $6 \mathrm{~mm}$ and height $4 \mathrm{~mm}$ has been simulated in Maxwell 3D. Its magnetic field lines (Fig. 17(b)) are better contained inside the coil than for the solenoid.

For having a comparative figure of merit of the magnetic field emissions of the two inductors, the magnetic induction along the $Z$-axis was simulated (Fig. 18). The magnetic induction of the toroid resulted 10 times lower than the one of the solenoid close to the inductor, and even lower as the distance increases.

\section{Phase Node Consideration and Shielding}

The phase node ( $N_{\text {phase }}$ in Fig. 1$)$ has the highest time varying voltage $(\mathrm{dv} / \mathrm{dt})$ in the converter. Its surface must be kept as small as possible, in order to reduce the stray capacitance to ground plates outside the PCB. This is more critical in power converters like AC-DC half-bridge [11], where the switches are typically mounted in the heat sink through a thin electrical insulating material. The unavoidable stray capacitance between the switch and the heat sink (connected to

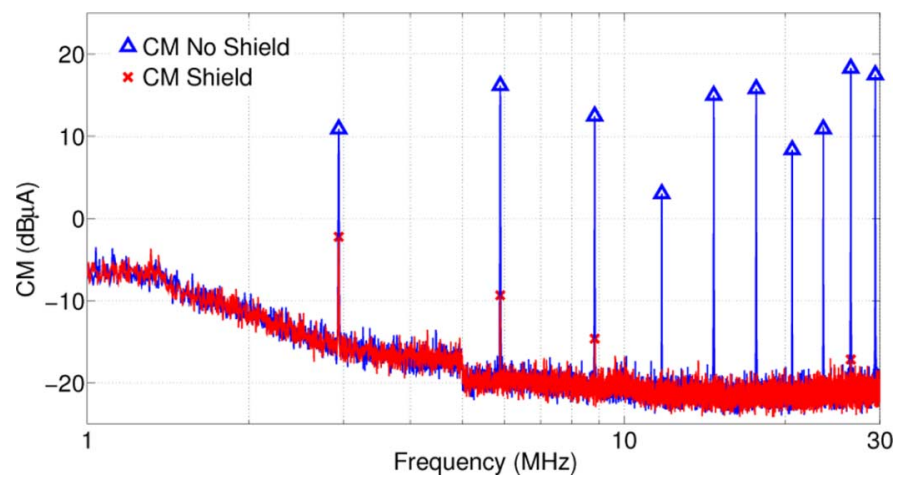

Fig. 19. CM current for a converter with or without shield.

ground for safety reasons) closes the loop for the generation of $\mathrm{CM}$ currents. Even if the switches dimensions (and therefore, the stray capacitance values) are several time smaller in our converter, this certainly still represents an issue.

A shield can be used to reduce capacitive coupling between the phase node and the ground plane external to the board. For that, a good electrical contact between shield and return path must be insured. For achieving this in our prototypes, three metallic clips were used as mechanic support for the shield and their pad electrically connected to the return layer of the PCB (as shown in the schematic of Fig. 10).

Fig. 19 shows the reduction achieved in the CM currents for a prototype working at $10 \mathrm{~V}$ after the addition of a $100 \mu \mathrm{m}$ thick copper shield. When the shield is not present ( $\triangle$ signal), the noise does not decrease with the frequency. This is due to the fact that the stray capacitances decrease their impedance with the increase of the frequency. The addition of the shield gives a new path to the current originated by the switching nodes, preventing the $\mathrm{CM}$ from being originated. As expected, the impact of the shield is higher as the frequency augments and attenuation of more than $10 \mathrm{~dB}$ can be reached. Nevertheless, the shield imposes a penalty to the material budget of the tracking detectors where the converters are going to be used (the mass of the tracker must be minimized, including the power converters used to power them).

\section{MEASUREMENTS AND RESULTS}

A $9 \mathrm{~W}$ converter prototype based on the simplified schematic of Fig. 10 was designed following the considerations described above. This buck converter (Fig. 20(a)) provides an output regulated voltage of $2.5 \mathrm{~V}$, for typical input voltages of 8-14 V. The nominal operation of the converter is $10 \mathrm{~V}$ at the input, 2.5 $\mathrm{V}$ and $1 \mathrm{~A}$ at the output with an efficiency of around $80 \%$. The switching frequency is $3 \mathrm{MHz}$, the main inductor has an inductance of $500 \mathrm{nH}$ and,therefore, a peak to peak current of $1.25 \mathrm{~A}$.

The noise of the shielded converter (Fig. 20(b)) was measured under nominal conditions at the measuring port of a line impedance stabilization network (LISN) [12]. The measurements were based on CISPR11 standard [13], using an EMI Test Receiver (R\&S ESPI3) with maximum peak detector (for achieving a worst-case condition).

The voltage at the Line port of the LISN, representing the voltage drop induced by sum of CM and DM currents on the 


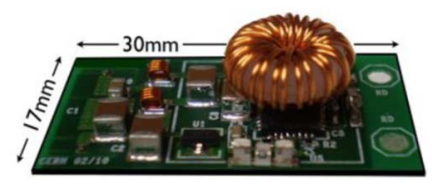

(a)

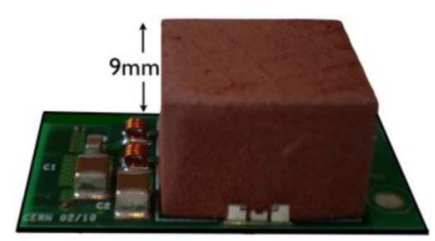

(b)
Fig. 20. Pictures of the buck converter prototype used for system level tests. (a) Converter without shield (b) Converter with shield.

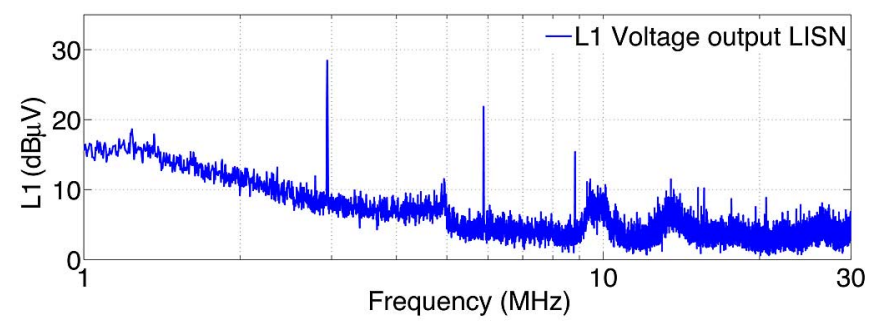

(a)

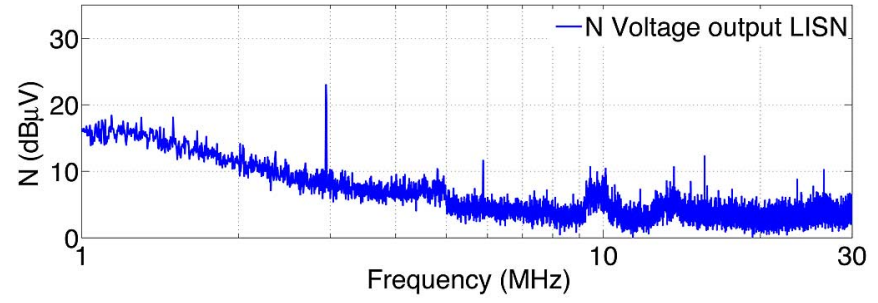

(b)

Fig. 21. Line and Neutral voltages output LISN. (a) L1 voltage output LISN (b) $N$ voltage output LISN.

$50 \Omega$ LISN impedance $\left(V_{L 1}=\left(I_{c m}+I_{d m}\right) \cdot 50 \Omega\right)$, is shown in Fig. 21(a). Just three harmonics overhang the noise floor of the EMI receiver, with a maximum value for the fundamental of $28 \mathrm{~dB} \mu \mathrm{V}(25 \mu \mathrm{V})$. Similarly, the voltage on the Neutral port of the LISN (corresponding to the difference of CM and DM currents times the LISN impedance, $V_{N}=\left(I_{c m}-I_{d m}\right) \cdot 50 \Omega$ ) was measured. The Neutral voltage $\left(V_{N}\right)$ has a maximum value of $23 \mathrm{~dB} \mu \mathrm{V}(14 \mu \mathrm{V})$ for the fundamental frequency as shown in Fig. 21(b).

The measured noise performance, compatible with class B of CISPR11 standard [13], is considered sufficiently low to try powering representative detector modules for the LHC upgrades. This test should evidence if the use of DCDC converters is compatible with the strict noise requirements of the aimed application.

\section{A. Compatibility Test With an ATLAS Tracker Module}

A prototype module for the short-strip detector of the ATLAS HLLHC [14] is used for the compatibility test. A single module (Fig. 22) consists of two silicon microstrip sensors and four kapton multi-layer hybrid flex circuits, arranged in two sides. Each hybrid holds two rows of 10 ABCN FE ASICs [15] of 128 readout channels bonded to a strip of the silicon sensor for readout purpose.

For evaluating the performance of the module, the FE ASIC has an internal calibration circuit which allows the simulation

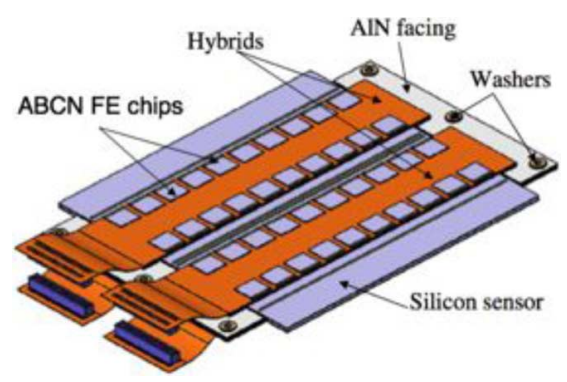

Fig. 22. Single double-sided silicon microstrip module concept. (Picture courtesy of $S$. Gonzalez-Sevilla, University of Geneva).

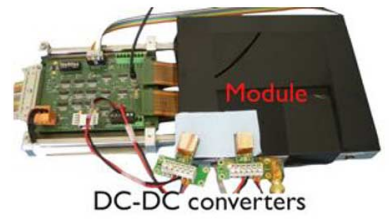

(a)

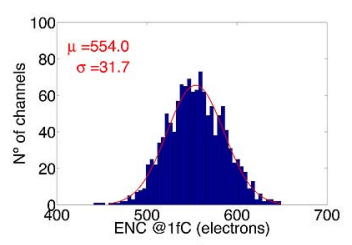

(c)

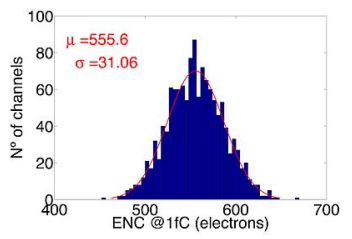

(b)

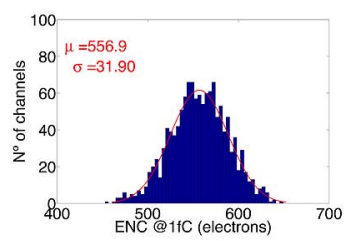

(d)
Fig. 23. Equivalent input noise of a single module for different powering configurations. (a) Module powered (b) ENC: powered by Linear PS (c) ENC: powered by converter (d) ENC: powered as shown in (a).

of a hit in a strip by the injection of test charges of selectable amplitude. Through a three point gain scan [16] it is possible to obtain the corresponding input noise (Equivalent Noise Charge or ENC) as a function of channel number. The compatibility between a converter and a module can be considered achieved if the input noise of a module powered by the converter is the same as the one obtained when power is provided by a linear power supply. Fig. 23(b) shows the equivalent input noise obtained for a row of ten ASICs when powering a module prototype of the University of Geneva (Fig. 23(a)) with a linear power supply. The mean ENC value $(\mu)$ is close to 556 electrons at $1 \mathrm{fC}$ of injected charge.

Afterwards, the equivalent input noise of the module was measured in the same conditions with power provided by DC-DC converter prototypes. Two converters (Fig. 20(b)) were needed as this module uses separated power inputs for analog and digital circuitry (1 A and $3 \mathrm{~A}$ respectively). Those converters were placed more than $15 \mathrm{~cm}$ far from the hybrid in order to prevent electromagnetic couplings. Any deterioration of the module performance would hence be attributed to conductive noise from the converter. The obtained input noise of 554 electrons (Fig. 23(c)) is identical to the one measured with linear power supply.

Because of the extremely compact design of the real strip detector implementation, converters will necessarily be placed much closer to the module and electromagnetic couplings could 
potentially affect the performance. For this reason, measurements were repeated with the converters put at $2 \mathrm{~cm}$ from the module (as shown in Fig. 23(a)), on top of a black plastic cover that prevented the light from reaching the silicon strips and disturb the measurements. In such a close proximity ("worst case"), electromagnetic coupling with the FE ASIC and sensor could affect and impair the performance of the module. Nevertheless, the equivalent input noise did not change, as shown in Fig. 23, confirming the full EMI compatibility of the developed converter.

\section{CONCLUSION}

The LHC upgrade (HLLHC) requires a new powering scheme for the electronics inside the experiments. One possible solution is based on DC-DC converters mounted on the FE modules, which must be radiation and magnetic field tolerant. These switching converters intrinsically emit EMI that may degrade the performance of the FEE. Along this paper, different design strategies for reducing the emitted noise were proposed.

The reduction of emissions for all or some harmonics was proved to be possible knowing the sources of noise. This is particularly important if the FEE is known to be sensitive to EMI in a certain frequency range.

Non-idealities of components and board traces have negative impact in emissions, which get stronger as the frequency augments. Similarly, EM couplings between components may impair the performance of the filters. Careful selection and segregation of the components are strategies that, done at an early stage, can help to decrease considerably the EM emissions.

Converter prototypes, implemented following the above design strategies, were used to power a short-strip module prototype for the ATLAS HLLHC upgrade. The performance of the system was not affected while powering with DC-DC converters, even when placed at close proximity ("worst case"). DC-DC converters represent a feasible solution for powering FE electronics while appropriate precautions are taken.

\section{REFERENCES}

[1] M. Weber, "Power distribution for SLHC trackers: Challenges and solutions," Nucl. Instrum. Methods Phys. Res. A, vol. 592, pp. 44-55, 2008.

[2] C. Fuentes et al., "Power distribution with custom DC-DC converters for SLHC trackers," in Proc. Nuclear Science Symp., Oct. 2009.

[3] F. Faccio et al., "TID and displacement damage effects in vertical and lateral power MOSFETs for integrated DC-DC converters," IEEE Trans. Nucl. Sci., vol. 57, no. 4, pp. 1790-1797, Aug. 2010.

[4] S. Orlandi et al., "Optimization of shielded pcb air-core toroids for high efficiency DC-DC converters," IEEE Trans. Power Electron., 2011, IEEE Xplore website.

[5] E. Hoene et al., "Simulating electromagnetic interactions in high power density inverters," in Proc. IEEE 36th Power Electronics Specialists Conf., 2005, pp. 1665-1670.

[6] S. Michelis et al., "An 8w-2 mhz buck converter with adaptive dead time tolerant to radiation and high magnetic field," in Proc. ESSCIRC Conf., Sevilla, Spain, Sep. 2010.

[7] H. W. Ott, Electromagnetic Compatibility Engineering. New York: Wiley, 2009.

[8] C. Holloway and E. Kuester, "Net and partial inductance of a microstrip ground plane," IEEE Trans. Electromagn. Compat., vol. 40, no. 1, pp. 33-46, Feb. 1998.

[9] A. Lissner, E. Hoene, B. Stube, and S. Guttowski, "Predicting the influence of placement of passive components on EMI behaviour," in Proc. Euro. Conf. Power Electronics and Applications, 2007, pp. 1-10.

[10] K. Armstrong, EMC for Printed Circuit Boards: Basic and Advanced Design and Layout Techniques. Cornwall, U.K.: Nutwood, 2007.

[11] M. Jin et al., "Identification of essential coupling path models for conducted EMI prediction in switching power converters," IEEE Trans. Power Electron., vol. 21, no. 6, pp. 1795-1803, Nov. 2006.

[12] CISPR 16-1-2: Specification for Radio Disturbance and Immunity Measuring Apparatus and Methods-Radio Disturbance and Immunity Measuring Apparatus: Ancillary Equipment-Conducted Disturbances, IEC, 2006.

[13] CISPR 11: Industrial, Scientific and Medical (ISM) Radio-Frequency Equipment-Electromagnetic Disturbance Characteristics-Limits and Methods of Measurement, IEC, 2003.

[14] S. Gonzalez-Sevilla et al., "A silicon strip module for the ATLAS inner detector upgrade in the super LHC collider," Nucl. Instrum. Methods Phys. Res. A, vol. 636, pp. S97-S103, 2011.

[15] J. Kaplon et al., "The ABCN front-end chip for ATLAS inner detector upgrade," in Proc. Topical Workshop Electronics for Particle Physics, Naxos, Greece, 2008, pp. 116-120.

[16] P. W. Phillips, "Functional testing of the ATLAS SCT barrels," Nucl Instrum. Methods Phys. Res. A, vol. 570, pp. 230-235, 2007. 